\title{
SENSE OF COHERENCE IS SIGNIFICANTLY ASSOCIATED WITH BOTH METABOLIC SYNDROME AND LIFESTYLE IN JAPANESE COMPUTER SOFTWARE OFFICE WORKERS
}

\section{YUSAKU MORITA, MASANORI OHTA, TOMOHIRO INOUE, TORU HONDA, YOSHIMASA KONNO, YASUMASA EGUCHI, and HIROSHI YAMATO}

University of Occupational and Environmental Health, Japan, Kitakyushu City, Fukuoka, Japan

Institute of Industrial Ecological Sciences, Department of Health Development

\begin{abstract}
Objectives: Sense of coherence (SOC) is an individual characteristic related to a positive life orientation, leading to effective coping. Little is known about the relationship between SOC and metabolic syndrome (MetS). This cross-sectional study aimed at testing the hypothesis that workers with a strong SOC have fewer atherosclerotic risk factors, including MetS, and healthier lifestyle behaviors. Material and Methods: One hundred and sixty-seven computer software workers aged 20-64 years underwent a periodical health examination including assessment of body mass index, waist circumference, blood pressure, blood lipid levels, fasting blood sugar (FBS) levels and lifestyle behaviors (walking duration, smoking status, nutrition, alcohol consumption, and sleep duration). During this period, the participants also completed a 29-item questionnaire of SOC and the Brief Job Stress Questionnaire to assess job stressors such as job strain and workplace social support. Results: Our results showed that the participants with a stronger SOC were likely to walk for at least $1 \mathrm{~h}$ a day, to eat slowly or at a moderate speed, and to sleep for at least $6 \mathrm{~h}$. Compared with the participants with the weakest SOC, those with the strongest SOC had a significantly lower odds ratio (OR) for being overweight (OR $=0.31 ; 95 \%$ confidence interval (CI): 0.11-0.81), and having higher FBS levels (OR = 0.11; 95\% CI: 0.02-0.54), dyslipidemia (OR = 0.29; 95\% CI: 0.09-0.84), and MetS (OR $=0.12$; 95\% CI: 0.02-0.63), even after adjusting for age, gender and job stressors. Conclusions: High SOC is associated with a healthy lifestyle and fewer atherosclerotic risk factors, including MetS.
\end{abstract}

Key words:

Metabolic syndrome, Lifestyle, Sense of coherence, Job stressor, Atherosclerotic risk factors

\section{INTRODUCTION}

Ischemic heart disease and cerebrovascular disease are the leading causes of death in Japan. The Ministry of Health, Labour and Welfare reported that in 2011 [1], approximately 78000 deaths were caused by ischemic heart disease and approximately 124000 deaths by cerebrovascular disease. Both diseases are caused by atherosclerosis, which has a number of risk factors including elevated blood sugar levels, elevated blood pressure and dyslipidemia.

Presence of multiple risk factors significantly increases the likelihood of developing atherosclerosis [2]. Metabolic syndrome (MetS) represents an accumulation of atherosclerotic risk factors. A previous study reported

Received: March 19, 2014. Accepted: July 18, 2014.

Corresponding author: Y. Morita, University of Occupational and Environmental Health, Japan, Institute of Industrial Ecological Sciences, Department of Health Development, 1-1 Iseigaoka, Yahatanishi-ku, Kitakyushu 807-8555, Japan (e-mail: morita@med.uoeh-u.ac.jp). 
a significant reduction in the prevalence of MetS through lifestyle intervention [3], and it is now clear that a healthy lifestyle is important for improving MetS. In 2008, "Health Check-ups and Healthcare Advice with a Particular Focus on the Metabolic Syndrome (MetS)," which is a health care guide aimed at improving the dietary and exercise habits of patients, was provided by physicians or public health nurses in order to reduce the occurrence of cerebrovascular and ischemic heart diseases. This initiative focused on tackling MetS.

The concept of the sense of coherence (SOC) was introduced by Aaron Antonovsky as part of the salutogenic theory, which examines why some people encountering stressors and major life events remain healthy, while others do not [4]. A strong SOC shows that an individual believes that what happens in their lives is rational, predictable, structured and understandable (comprehensibility); that they have a perception that adequate and sufficient resources are available to help resolve difficulties as they arise (manageability); and a view that the demands created by exposure to adversity are challenges and worthy of engagement (meaningfulness).

A recent review noted that SOC is a health promoting resource that strengthens resilience and develops a positive subjective state of health. The review showed that there are positive associations between a strong SOC and both mental and physical health [5]. The Helsinki heart study has shown that the lowest incidence of coronary heart disease was found in the strongest SOC quintile [6]. Additionally, SOC has also been shown to be associated with healthy lifestyle choices [7].

To our knowledge, there are no published studies demonstrating an association between SOC and both multiple atherosclerotic risk factors, including MetS, and lifestyle behaviors in the same subjects. However, there are a few studies that have shown an association between SOC and objective physical health $[8,9]$. We aimed at examination of the hypothesis that a strong SOC, in Japanese computer software office workers, is associated with both healthy lifestyle behaviors and reduced atherosclerotic risk factors, including MetS.

\section{MATERIAL AND METHODS}

We conducted a cross-sectional study among white-collar workers of a Japanese computer software company that had 185 employees (144 men and 41 women). One hundred and sixty-seven employees (130 men and 37 women, approximately $90 \%$ of all the employees), aged 20-64 years, participated in our study and all of them underwent a periodical health examination.

The other 18 employees' lifestyle-related information was not available because they underwent health check-ups in other institutions, which did not use our lifestyle-related questionnaires, and therefore, they were excluded. During the same period as the health examination, a self-administered questionnaire regarding SOC and job stressors was distributed to each employee through the inter-office e-mail. They confidentially returned completed questionnaires by e-mail, and not via their managers or employer. Before conducting the study, we explained its design and purpose to a safety and health committee consisting of representatives of the employers and employees. They agreed to this study, and the study's design and purpose were explained to all the employees by the committee. The study protected the participants' privacy, and participation in the survey was voluntary. We ensured that all the workers had the possibility to terminate their participation in the study at any time. The study was approved by the Ethics Committee of the University of Occupational and Environmental Health, Japan.

\section{Periodical health examination}

In May 2011, 167 workers underwent a periodical health examination and filled in a self-administered questionnaire to assess their lifestyle behaviors at their place of work. In addition to the questionnaire, during the periodical 
health examination we recorded the following data: age, gender, body mass index (BMI, calculated from height and weight), waist circumference (for those aged 35 years and older), blood pressure (systolic blood pressure - SBP; diastolic blood pressure - DBP), as well as fasting blood sugar (FBS) levels, triglyceride (TG) levels, low-density lipoprotein cholesterol (LDL-C) levels and high-density lipoprotein cholesterol (HDL-C) levels.

Blood samples were collected after at least $9 \mathrm{~h}$ of fasting. Waist circumference was measured only in the case of the participants aged $\geq 35$ years $(\mathrm{N}=126)$, and that was in line with the legal requirements placed upon employers to conduct periodic health screening; hence, the presence of MetS was also assessed only in 126 participants (110 men and 16 women).

\section{Anthropometric measurements and blood examinations}

Anthropometric measurements and the results of blood tests were divided into 2 different groups based on the following MetS criteria (Table 1). Body mass index $\left(\mathrm{kg} / \mathrm{m}^{2}\right) \geq 25$ was categorized as overweight and BMI $<25$ as normal; waist circumference, if $\geq 85 \mathrm{~cm}$ for men and $\geq 90 \mathrm{~cm}$ for women was categorized as abdominal obesity, or normal if the waist circumference was lower than the limits mentioned above. Blood pressure was categorized as elevated if SBP $\geq 130$ and/or DBP $\geq 85 \mathrm{~mm} \mathrm{Hg}$, and/or in the case of hypertension treatment or as normal for lower pressures and no hypertension.

For biochemical markers, FBS was categorized as elevated if it was higher or equalled $110 \mathrm{mg} / \mathrm{dl}$ and/or in the case of diabetes treatment, or as normal in the case of lower blood sugar levels and no diabetes. Biomarkers of lipid metabolism (HDL-C and TG) were categorized as dyslipidemia (HDL-C level < $40 \mathrm{mg} / \mathrm{dl}$ and/or TG $\geq 150 \mathrm{mg} / \mathrm{dl}$ and/ or medication of dyslipidemia) or as normal (no elevated marker levels and no medication). Diagnosis of MetS was performed in accordance with the definition of the Examination Committee of Criteria for the Metabolic Syndrome in Japan (Table 1) [10]. With the subjects who took specific medications for the components examined during the diagnosis, each medication was considered a positive component for the diagnosis.

Table 1. Current diagnostic criteria of the metabolic syndrome in Japan

\begin{tabular}{lc}
\hline \multicolumn{1}{c}{ Symptom } & Criteria \\
\hline Abdominal obesity - waist circumference $(\mathrm{cm})$ & \\
men & $\geq 85$ \\
women & $\geq 90$ \\
Risk factor (at least 2$)$ & $\geq 150$ \\
elevated triglyceride level $(\mathrm{mg} / \mathrm{dl})$ & $<40$ \\
reduced HDL-C level $(\mathrm{mg} / \mathrm{dl})$ & \\
elevated blood pressure $(\mathrm{mm} \mathrm{Hg})$ & $\geq 130$ \\
$\quad$ systolic pressure & $\geq 85$ \\
$\quad$ dyastolic pressure & $\geq 110$ \\
elevated blood sugar level $(\mathrm{mg} / \mathrm{dl})$ & \\
\hline
\end{tabular}

HDL-C - high-density lipoprotein cholesterol.

\section{Lifestyle-related questionnaire}

We assessed 5 lifestyle behaviors: smoking habit, alcohol consumption, walking habit, sleep duration and rate of eating. Selection of these lifestyle factors was based on the results of the previous studies that had investigated their relationship with obesity or mortality [11-15].

Smoking habit was divided into 3 categories: "non-smoker" (never smoked), "ex-smoker," and "current smoker." Alcohol consumption was firstly divided into "currently drinking" and "currently not drinking" categories, and subsequently, current drinkers were asked about their mean daily consumption of alcohol based on the traditional Japanese unit of alcohol beverage - the gou ( 1 gou $=180 \mathrm{ml}$ of sake, a traditional Japanese rice wine, containing $22 \mathrm{~g}$ alcohol). On this basis, alcohol consumption was divided into "less than 1 gou," "1 to 2 gou," "2 to 3 gou," and "more than 3 gou." Walking habits were addressed with the question "Do you walk every day 1 hour or more?" (yes/no). Sleep duration 
was classified into 6 categories based on the self-reported responses: less than $3 \mathrm{~h} ; 4,5,6$, and $7 \mathrm{~h}$; and more than $7 \mathrm{~h}$. Rate of eating was self-reported according to 1 of the following 3 categories: "slow," "moderate," and "fast."

Each lifestyle was classified into 2 categories based on these values: for smoking - "current smoker" and "others" [14]; for alcohol consumption - "drinking no more than 1 gou per day" describing those drinking no more than 1 gou or not drinking at all, and "others" [11]; for walking - "walking no less than 1 hour every day" and "others" [13]; for sleep duration - "sleeping no less than 6 hours" and "others" [12]; and for the rate of eating - "eating fast" and "not eating fast (moderate or slow)" [15].

\section{Sense of coherence}

Sense of coherence was originally measured by 29 questions that were answered using a 7-point Likert scale. The scale has:

- 11 items on comprehensibility - e.g., "Do you have very mixed-up feelings and ideas?," scored from 1 (very often) to 7 (very rarely or never),

- 10 on manageability - e.g., "Has it ever happened that people who you counted on disappointed you?," scored from 1 (never happened) to 7 (always happens),

- 8 on meaningfulness - e.g., "Do you have the feeling that you don't really care about what goes on around you?," scored from 1 (very seldom or never) to 7 (very often).

A high score indicates a strong SOC. It has been used in the actual SOC questionnaire, and the validity of the questionnaire has been previously examined [16]. In this study, we used the Japanese version of the SOC questionnaire translated by Yamazaki [17]. For analysis, the summary score was divided into tertiles indicating a weak, medium, or strong SOC: a score of $\geq 130$ points was defined as a strong SOC, a score of $\geq 113$ but $<130$ was defined as a medium SOC, and a score of $<113$ points was defined as a weak SOC.

\section{Job stressors}

Job stressors were assessed using the Brief Job Stress Questionnaire (BJSQ), which was developed and validated by the Ministry of Health, Labour and Welfare of Japan $[18,19]$. The BJSQ is a multi-dimensional questionnaire with 4-point Likert scale response options (1 - "strongly disagree" to 4 - "strongly agree") to measure job stressors. Quantitative job demand, decision latitude, co-workers' support and supervisors' support are the job stressors measured by the BJSQ, which is essentially based on the demand-control-support model [20]. All of the BJSQ scales have acceptable levels of internal consistency, reliability and factor-based validity [21,22].

Based on the methodology used in previous studies, we calculated job strain by dividing quantitative job demand by decision latitude [23,24], and workplace social support was calculated as the sum of co-workers' support and supervisors' support $[18,25]$.

\section{Job status}

The participants' job status was obtained from personnel files and categorized as either managerial or general.

\section{Statistical analysis}

We assessed the statistical significance of continuous variables using ANOVA, and differences between the groups were evaluated with a post hoc Tukey test (an $\alpha$ value of 0.05 was adopted). For categorical variables, we used $\mathrm{Chi}^{2}$ tests, and linear trends were verified using the Cochran-Armitage trend test.

In order to avoid medication effects when analyzing blood pressure, lipid profile and fasting blood glucose, we excluded data from the participants who were undergoing specific treatments for hypertension, dyslipidemia and diabetes. The multivariate logistic regression analysis was used to estimate the association between SOC or lifestyle behaviors and atherosclerotic risk factors after adjusting for age, gender, job strain, workplace social support and job status. 
All statistical calculations were performed with the JMP statistical software package (JMP 9.0; SAS Institute Inc., Cary, NC, USA). The results of continuous variables are expressed as a mean \pm standard deviation. Values of $p<0.05$ were considered as statistically significant.

\section{RESULTS}

\section{Characteristics of the participants}

The mean age of the participants was $41.2 \pm 9.8$ years (43.1 \pm 8.8 years in men and $34.6 \pm 10.3$ years in women). There were 29 managers (27 men and 2 women). The mean SOC score was $120.7 \pm 21.0$ points $(121.4 \pm 21.8$ points in men and $118.2 \pm 18.1$ points in women) with a Cronbach's $\alpha$ coefficient of 0.92 . The response rate of the questionnaire was 100\% (completed by all the 167 participants).

\section{Association between SOC and lifestyle behaviors}

The prevalence of the participant's lifestyles with respect to the 3 SOC levels was shown in Table 2. The participants with a stronger SOC were more likely to walk for at least $1 \mathrm{~h}$ per day ( $\mathrm{p}$ for trend $=0.04$ ), to sleep for no less than $6 \mathrm{~h}$ each day ( $\mathrm{p}$ for trend $=0.02$ ), and were less likely to eat fast ( $p$ for trend $=0.02$ ).

\section{Association between SOC and job stressors or common atherosclerotic risk factors}

There were no significant differences in age or gender distribution between the different SOC groups. The participants with a strong SOC had a significantly lower BMI and

Table 2. Association between the sense of coherence (SOC) and lifestyle

\begin{tabular}{|c|c|c|c|c|c|}
\hline \multirow[t]{2}{*}{ Category } & \multicolumn{3}{|c|}{$\begin{array}{c}\text { Respondents } \\
(\mathrm{N}=167) \\
{[\mathrm{n}(\%)]}\end{array}$} & \multirow[t]{2}{*}{$\mathrm{p}$} & \multirow[t]{2}{*}{$\mathrm{p}$ for trend } \\
\hline & weak & medium & strong & & \\
\hline \multicolumn{6}{|l|}{ Walking duration } \\
\hline$\geq 1 \mathrm{~h} /$ day & $51(89.5)$ & $51(91.1)$ & $41(75.9)$ & $0.046^{*}$ & $0.04^{*}$ \\
\hline$<1$ h/day & $6(10.5)$ & $5(8.9)$ & $13(24.1)$ & & \\
\hline \multicolumn{6}{|l|}{ Rate of eating } \\
\hline not fast & $28(49.1)$ & $19(33.9)$ & $15(27.8)$ & 0.06 & $0.02^{*}$ \\
\hline fast & $29(50.9)$ & $37(66.1)$ & $39(72.2)$ & & \\
\hline \multicolumn{6}{|l|}{ Smoking habit } \\
\hline current smoker & $11(19.3)$ & $17(30.4)$ & $12(22.2)$ & 0.36 & 0.7 \\
\hline non- and ex-smoker & $46(80.7)$ & $39(69.6)$ & $42(77.8)$ & & \\
\hline \multicolumn{6}{|l|}{ Alcohol consumption } \\
\hline$<1$ gou/day & $18(31.6)$ & $19(33.9)$ & $20(37.0)$ & 0.83 & 0.54 \\
\hline$\geq 1$ gou/day & $39(68.4)$ & $37(66.1)$ & $34(63.0)$ & & \\
\hline \multicolumn{6}{|l|}{ Sleeping duration } \\
\hline$\geq 6 \mathrm{~h} /$ day & $31(54.4)$ & $40(71.4)$ & $41(75.9)$ & $0.04^{*}$ & $0.02^{*}$ \\
\hline$<6 \mathrm{~h} /$ day & $26(45.6)$ & $16(28.6)$ & $13(24.1)$ & & \\
\hline
\end{tabular}

$\mathrm{Chi}^{2}$ tests were used and linear trends were verified using the Cochran-Armitage trend test SOC.

$1 \mathrm{gou}=22 \mathrm{~g}$ alcohol $(180 \mathrm{ml}$ of traditional Japanese rice wine $)$.

$* \mathrm{p}<0.05$. 
waist circumference than those with a weak SOC. The participants with a weak SOC suffered significantly more from work-related stress and received lesser support at work than the participants who had a medium or strong SOC. The number of participants undergoing hypertension, dyslipidemia, and diabetes treatments was not significantly different. The blood sugar level in the strong SOC group was significantly lower than in the medium SOC group. However, there were no significant differences in blood pressure and lipid profiles between the groups (Table 3).
After adjusting for age, gender, job stressors and job status, the strong SOC group had a significantly lower odds ratio $(\mathrm{OR})$ for being overweight $(\mathrm{OR}=0.31$; 95\% CI: 0.11-0.81), dyslipidemia $(\mathrm{OR}=0.29$; 95\% CI: 0.09-0.84), elevated blood sugar levels $(\mathrm{OR}=0.11 ; 95 \%$ CI: 0.02-0.54) and MetS (OR = 0.12; 95\% CI: 0.02-0.63). The medium SOC group had a significantly lower $\mathrm{OR}$ for being overweight $(\mathrm{OR}=0.37$; 95\% CI: 0.15-0.89) and abdominal obesity (OR $=0.35$; 95\% CI: 0.12-0.96) (Table 4).

Table 3. Association between the sense of coherence (SOC) and job stressors or atherosclerotic risk factors

\begin{tabular}{|c|c|c|c|c|}
\hline \multirow{2}{*}{ Criterium } & \multicolumn{4}{|c|}{ Sense of coherence } \\
\hline & weak & medium & strong & $\mathrm{p}$ \\
\hline Age (years) $(\mathrm{M} \pm \mathrm{SD})$ & $39.5 \pm 8.3$ & $42.6 \pm 10.9$ & $41.5 \pm 9.9$ & 0.25 \\
\hline Male/female (n) & $42 / 15$ & $44 / 12$ & $44 / 10$ & 0.61 \\
\hline Managerial/general (n) & $7 / 50$ & $6 / 50$ & $16 / 38$ & $0.01^{*}$ \\
\hline Job strain ${ }^{\mathrm{a}}(\mathrm{M} \pm \mathrm{SD})$ & $1.45 \pm 0.67$ & $1.12 \pm 0.46^{\mathrm{f}}$ & $0.96 \pm 0.33^{\mathrm{f}}$ & $<0.001^{*}$ \\
\hline Workplace social support $\mathrm{t}^{\mathrm{b}}(\mathrm{M} \pm \mathrm{SD})$ & $13.3 \pm 3.6$ & $15.1 \pm 2.7^{\mathrm{f}}$ & $16.8 \pm 3.2^{\mathrm{f}, \mathrm{g}}$ & $<0.001^{*}$ \\
\hline BMI & $25.2 \pm 6.3$ & $23.3 \pm 3.3$ & $22.9 \pm 3.3^{\mathrm{f}}$ & $0.01^{*}$ \\
\hline respondents (n) & 57 & 56 & 54 & \\
\hline Waist circumference $(\mathrm{cm})(\mathrm{M} \pm \mathrm{SD})$ & $87.7 \pm 14.8$ & $81.8 \pm 8.8^{\mathrm{f}}$ & $81.6 \pm 7.5^{\mathrm{f}}$ & $0.02^{*}$ \\
\hline respondents (n) & 41 & 43 & 42 & \\
\hline Hypertention treatment $(+/-)$ & $5 / 52$ & $8 / 48$ & $5 / 49$ & 0.59 \\
\hline $\mathrm{SBP}^{\mathrm{c}}(\mathrm{M} \pm \mathrm{SD})$ & $121.8 \pm 15.6$ & $123.2 \pm 14.0$ & $119.7 \pm 14.9$ & 0.52 \\
\hline $\mathrm{DBP}^{\mathrm{c}}(\mathrm{M} \pm \mathrm{SD})$ & $76.5 \pm 12.9$ & $75.3 \pm 13.0$ & $73.8 \pm 12.1$ & 0.56 \\
\hline Dyslipidemia treatment $(+/-)$ & $5 / 52$ & $6 / 50$ & $1 / 53$ & 0.17 \\
\hline $\mathrm{TG}^{\mathrm{d}}(\mathrm{M} \pm \mathrm{SD})$ & $112.9 \pm 63.5$ & $117.0 \pm 70.8$ & $118.8 \pm 97.6$ & 0.93 \\
\hline $\mathrm{HDL}^{\mathrm{d}}(\mathrm{M} \pm \mathrm{SD})$ & $59.9 \pm 16.8$ & $62.3 \pm 13.8$ & $62.7 \pm 16.8$ & 0.64 \\
\hline $\mathrm{LDL}^{\mathrm{d}}(\mathrm{M} \pm \mathrm{SD})$ & $125.2 \pm 32.0$ & $127.4 \pm 29.8$ & $120.4 \pm 28.3$ & 0.48 \\
\hline Diabetes treatment $(+/-)$ & $3 / 54$ & $4 / 52$ & $2 / 52$ & 0.73 \\
\hline $\mathrm{FBS}^{\mathrm{e}}(\mathrm{M} \pm \mathrm{SD})$ & $93.4 \pm 11.6$ & $96.0 \pm 9.8$ & $90.6 \pm 7.9^{\mathrm{g}}$ & $0.02 *$ \\
\hline
\end{tabular}

Statistical significance of categorical variables assessed using $\mathrm{Chi}^{2}$ tests.

Statistical significance of continuous variables assesed using 1-way ANOVA followed by a post hoc Tukey test.

Job stressors (job strain and workplace social supports) assessed using the Brief Job Stress Questionnaire.

${ }^{a}$ Calculated by dividing quantitative job demand by decision latitude; ${ }^{b}$ Calculated as the sum of supervisor support and co-worker support; ${ }^{\mathrm{c}}$ Data from participant with medication for hypertension were excluded; ${ }^{\mathrm{d}}$ Data from participant with medication for dyslipidemia were excluded; ${ }^{\mathrm{C}} \mathrm{Data}$ from participant with medication for diabetes were excluded; ${ }^{\mathrm{f}} \mathrm{p}<0.05$ compared to weak SOC group; ${ }^{\mathrm{g}} \mathrm{p}<0.05$ compared to medium SOC group. SBP - systolic blood pressure; DBP - diastolic blood pressure; TG - triglyceride; HDL - high density lipoprotein cholesterol; LDL - low density lipoprotein cholesterol; FBS - fasting blood sugar. $* \mathrm{p}<0.05$. 


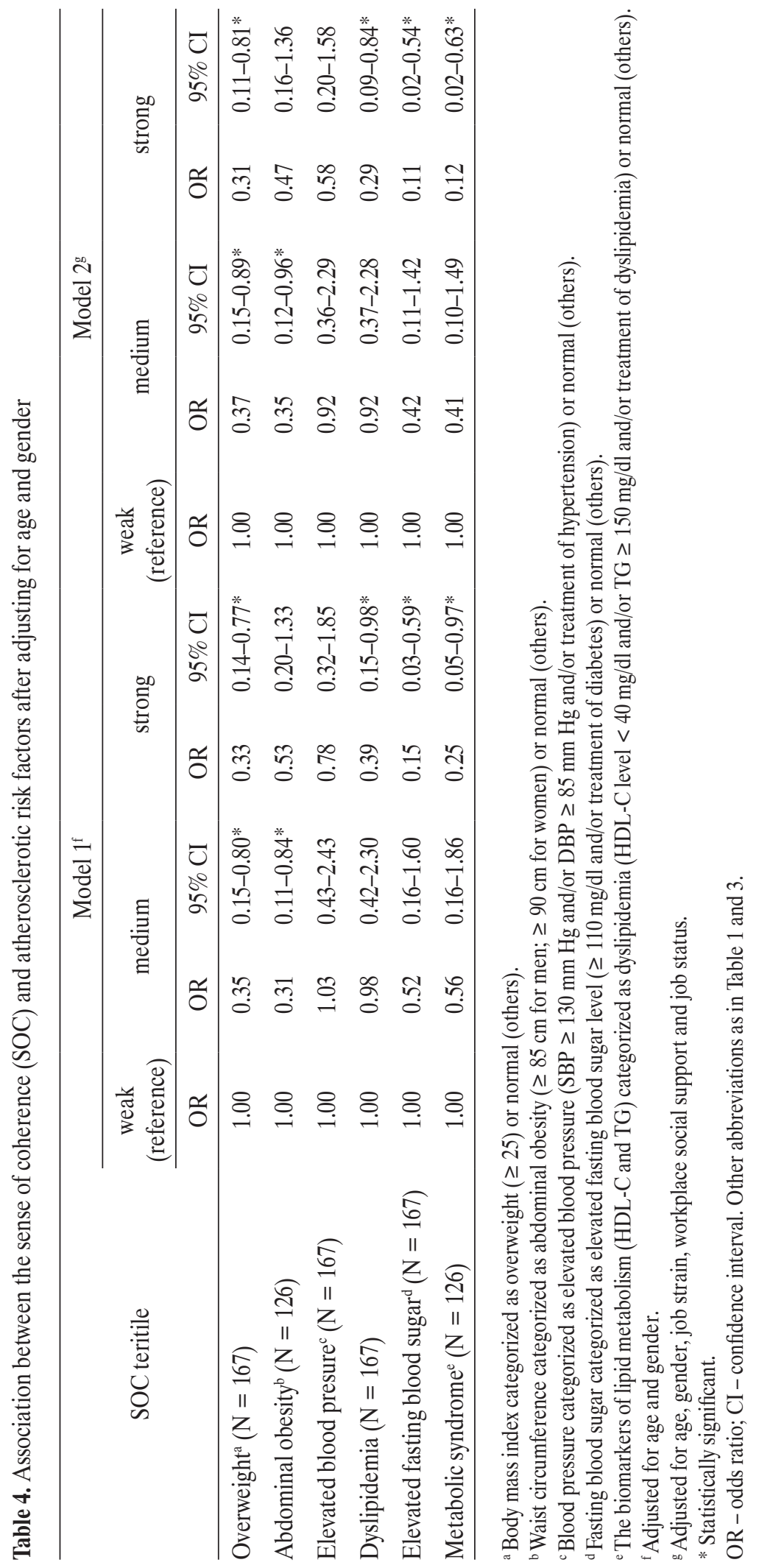




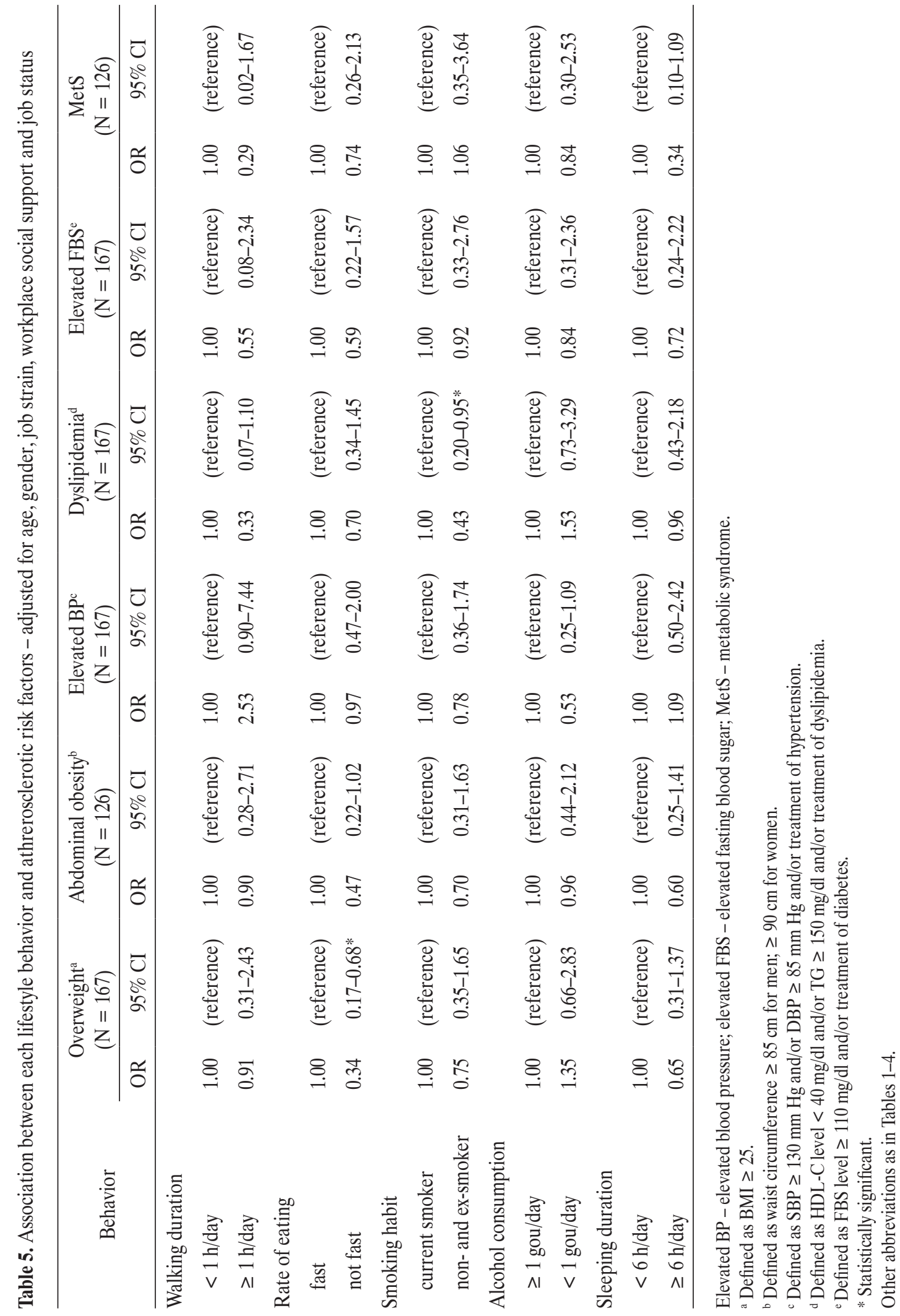




\section{Association between each lifestyle behavior and common atherosclerotic risk factors}

After adjusting for age, gender, job stressors and job status, it was found that the participants who ate slowly or at a moderate speed had a significantly lower OR for being overweight $(\mathrm{OR}=0.34 ; 95 \% \mathrm{CI}: 0.17-0.68)$, and that the participants who were non- or ex-smoker had a significantly lower OR for dyslipidemia (OR $=0.43$; 95\% CI: 0.19-0.95) (Table 5).

\section{DISCUSSION}

The results of our study support the hypothesis that a strong SOC is associated with reduced atherosclerotic risk factors as well as healthier lifestyle behaviors. This cross-sectional study suggests that Japanese computer software office workers who reported a strong SOC score were more likely to be physically active, more likely to sleep for no less than $6 \mathrm{~h}$ a night, less likely to eat quickly, less likely to be overweight as well as they were less likely to have elevated blood sugar levels, dyslipidemia and MetS.

The previous large-scale study in Europe (EPIC-Norfolk study) has also shown that strong SOC scores are associated with healthy lifestyle choices. Compared to those with the weakest SOC scores, those with the strongest scores were less likely to be current smokers and physically inactive, although, on average, they consumed more alcohol [26]. The present study also showed that the participants with a strong SOC were more likely to be physically active, although there was no association between SOC and smoking or alcohol consumption. Similarly, a study of 287 Finnish health care students has reported an association between SOC and physical exercise but not smoking or alcohol consumption [27].

Smoking rate in our study (approximately 24\%) was higher than that in the EPIC-Norfolk study cohort (11.8\% in the weak SOC group and 9.3\% in the strong SOC group). Alcohol consumption amongst our study participants (approximately 6.6 gou/week) was also higher than that amongst the EPIC-Norfolk study cohort (approximately 2.9 gou/week in the weak SOC group and 3.5 gou/week in the strong SOC group). It is possible that background information on the subjects with respect to culture, education and income level affected the association between SOC and smoking or alcohol consumption, although we did not collect those background data.

In addition, our results showed that the individuals with a strong SOC are more likely to sleep for at least $6 \mathrm{~h}$ a day. A number of research have reported an association between a strong SOC and sleep [28,29]. Taken together, these findings suggest that a strong SOC is associated with a healthy lifestyle.

Previous cross-sectional studies in female populations have reported an association between SOC and blood pressure [30], type 2 diabetes [31] and dyslipidemia [9]. The study in Japanese male, middle-aged workers has suggested that a weak SOC may be a risk factor for weight gain [8]. The present study also showed that SOC is associated with overweight, dyslipidemia and elevated blood sugar level.

In addition, our results indicate that SOC and MetS are negatively associated, suggesting that the individuals with a strong SOC are less likely to have risk factors for atherosclerosis. To the best of our knowledge, there are no published studies on the association between SOC and MetS, which is essentially an accumulation of atherosclerotic risk factors. Hence, we have provided new evidence for a relationship between SOC and MetS.

Cohen et al. have suggested a model to explain how stress affects health [32]. According to this model, when people face a stressful event, they perceive stress as if they had inadequate resources to resist the stressor. The perceived stress induces a negative emotionality that leads to psychophysiological and behavioral reactions, resulting in mental or physical disorders such as cardiovascular disease. 
There are at least 3 pathways that may explain the link between SOC and health. The 1st of these is toward a resistance resource. People with a weak SOC could perceive their environment as non-supportive [4]. Our results also suggested that a weak SOC is associated with low workplace social support. A previous report has suggested a moderating effect of SOC, whereby individuals with a strong SOC coped more efficiently with work environmental strain than those with a weak SOC [33]. People who perceived themselves to have little support may tend to have negative emotionality, resulting in poor health.

The 2nd pathway is toward negative emotionality. The SOC is strongly linked with aspects of negative emotionality [34], and negative emotionality in turn is associated with a higher BMI, weight gain [35] and MetS [36]. The 3rd pathway is toward stress coping behaviors. As Antonovsky has previously suggested [4], SOC influences effective implementation of health-related coping behavior.

The results we report here suggest that people with a strong SOC are less likely to eat fast. Additionally, there is an association between eating slowly or at a moderate speed and being overweight. A previous study has suggested that being overweight could lead to several lifestyle-related diseases such as diabetes, hypertension, dyslipidemia and MetS [37]. Furthermore, a previous prospective cohort study has reported that a strong SOC was associated with a $32 \%$ lower risk of diabetes [38], and another cohort study has reported that a strong SOC was associated with reduction in mortality from cardiovascular disease by 30\% [39]. It is possible then, that a strong SOC protects against atherosclerotic risk factors, in part through healthy lifestyle behaviors, and may result in a lower risk of death from a cardiovascular disease. It is also possible, though, that a weak SOC is a risk factor for MetS and is partly independent from the lifestyle. Therefore, occupational health staff should provide more generous support to health-care for those with a weak SOC, and this needs to be reflected in "Health Check-ups and Healthcare Advise with a Particular Focus on MetS" or any other health care guidance. The strengths of this study are the high response rate in the case of the questionnaire (100\%), the use of the full version of SOC scale (reliability and validity of the full version is better than the shorter version), and the measurement of atherosclerotic risk and lifestyle factors in the same subjects.

However, several potential limitations also have to be considered. First, since the analysis was cross-sectional, we were unable to draw conclusions on the causal relationship between SOC and lifestyle behaviors or atherosclerotic risk factors. This issue should be further examined by longitudinal studies.

Second, the sample size of this study is relatively small. It is possible that some associations may have been overlooked due to the lack of statistical power.

Third, we used a self-reported questionnaire to assess the participants' SOC and lifestyles. The individuals with a high SOC may sometimes report having a healthier lifestyle than they actually have. However, the present results also showed a relationship between SOC and objective health measures such as BMI and blood parameters, and thus, the relationship between SOC and the lifestyle-related parameters in the questionnaire may be relatively weak. Fourth, data on several confounding factors, including education and social class, were not available in this study. However, these participants' attributes may be relatively similar because all the participants were employees of the same company. Fifth, the SOC scores of our participants were slightly lower than those previously reported for Japanese populations ( $\mathrm{M} \pm \mathrm{SD}: 127.3 \pm 19.7$ ) [40].

Finally, all the participants were employees of the same computer software company, and generally did not undertake physically demanding work. Hence, the association between SOC and atherosclerotic risk factors might be different in blue-collar workers. Therefore, these findings may not be applicable to other companies or other populations. 


\section{CONCLUSIONS}

Our cross-sectional study on 167 computer software office workers suggested that a strong SOC is associated with reduced atherosclerotic risk factors, including MetS, and with healthier lifestyle behaviors with respect to walking, sleeping and dietary habit.

\section{REFERENCES}

1. [The Ministry of Health Law [Internet]. Summary of vital statistics; 2011] [cited 2014 March 5]. Available from: http:// www.mhlw.go.jp/toukei/saikin/hw/jinkou/kakutei11/index. html. Japanese.

2. Nakamura T, Tsubono Y, Kameda-Takemura K, Funahashi T, Yamashita S, Hisamichi S, et al. Magnitude of sustained multiple risk factors for ischemic heart disease in Japanese employees: A case-control study. Jpn Circ J. 2001;65(1):11-7, http://dx.doi.org/10.1253/jcj.65.11.

3. Ilanne-Parikka P, Eriksson JG, Lindström J, Peltonen M, Aunola S, Hämäläinen $\mathrm{H}$, et al. Effect of lifestyle intervention on the occurrence of metabolic syndrome and its components in the Finnish Diabetes Prevention Study. Diabetes Care. 2008;31(4):805-7, http://dx.doi.org/10.2337/dc07-1117.

4. Antonovsky A. Unraveling the mystery of health: How people manage stress and stay well. San Francisco: Jossey-Bass; 1987.

5. Eriksson M, Lindström B. Antonovsky's sense of coherence scale and the relation with health: A systematic review. J Epidemiol Community Health. 2006;60(5):376-81, http://dx.doi. org/10.1136/jech.2005.041616.

6. Poppius E, Tenkanen L, Kalimo R, Heinsalmi P. The sense of coherence, occupation and the risk of coronary heart disease in the Helsinki Heart Study. Soc Sci Med. 1999;49(1): 109-20, http://dx.doi.org/10.1016/S0277-9536(99)00105-7.

7. Wainwright NWJ, Surtees PG, Welch AA, Luben RN, Khaw KT, Bingham SA. Sense of coherence, lifestyle choices and mortality. J Epidemiol Community Health. 2008;62(9): 829-31, http://dx.doi.org/10.1136/jech.2007.066464.
8. Sagara T, Hitomi Y, Kambayashi Y, Hibino Y, Matsuzaki I, Sasahara S, et al. Common risk factors for changes in body weight and psychological well-being in Japanese male middle-aged workers. Environ Health Prev Med. 2009;14(6): 319-27, http://dx.doi.org/10.1007/s12199-009-0100-2.

9. Svartvik L, Lidfeldt J, Nerbrand C, Samsioe G, Scherstén B, Nilsson PM. Dyslipidaemia and impaired well-being in middle-aged women reporting low sense of coherence. The Women's Health in the Lund Area (WHLA) Study. Scand J Prim Health Care. 2000;18(3):177-82, http://dx.doi. org/10.1080/028134300453395.

10. Matuzawa Y, Ikeda Y, Katayama S, Kita T, Hisakiyama K, Saito $\mathrm{Y}$, et al. [Definition and diagnostic criteria of metabolic syndrome]. J Jpn Soc Inter Med. 2005;94(4):794-809. Japanese.

11. Lin Y, Kikuchi S, Tamakoshi A, Wakai K, Kawamura T, Iso $\mathrm{H}$, et al. Alcohol consumption and mortality among middle-aged and elderly Japanese men and women. Ann Epidemiol. 2005;15(8):590-7, http://dx.doi.org/10.1016/j.annepidem.2004.10.010.

12. Marshall NS, Glozier N, Grunstein RR. Is sleep duration related to obesity? A critical review of the epidemiological evidence. Sleep Med Rev. 2008;12(4):289-98, http://dx.doi. org/10.1016/j.smrv.2008.03.001.

13. Noda H, Iso H, Toyoshima H, Date C, Yamamoto A, Kikuchi $\mathrm{S}$, et al. Walking and sports participation and mortality from coronary heart disease and stroke. J Am Coll Cardiol. 2005;46(9):1761-7, http://dx.doi.org/10.1016/j.jacc. 2005.07.038.

14. Ozasa K. Smoking and mortality in the Japan Collaborative Cohort Study for Evaluation of Cancer (JACC). Asian Pac J Cancer Prev. 2007;8 Suppl:89-96.

15. Saito A, Kawai K, Yanagisawa M, Yokoyama H, Kuribayashi N, Sugimoto H, et al. Self-reported rate of eating is significantly associated with body mass index in Japanese patients with type 2 diabetes. Japan Diabetes Clinical Data Management Study Group (JDDM26). Appetite. 2012;59 (2):252-5, http://dx.doi.org/10.1016/j.appet.2012.05.009. 
16. Eriksson M, Lindström B. Validity of Antonovsky's sense of coherence scale: A systematic review. J Epidemiol Community Health. 2005;59(6):460-6, http://dx.doi.org/10.1136/ jech.2003.018085.

17. Yamazaki Y. [SOC, a theory on salutogenesis and health promoting ability from newly developed view points for health]. Quality Nursing. 1999;5:824-32. Japanese.

18. Kato M. [Study on prevention of work-related diseases. A research report concerning stress and its effects on health in workplace]. Ministry of Labour of Japan; 2000. p. 1-411. Japanese.

19. Japan Industrial Safety and Health Association [Internet]. [Guidance of stress check] [cited 2014 Feb 5]. Available from: http://www.jisha.or.jp/mental_health/stress_check.html. Japanese.

20. Johnson JV, Hall EM. Job strain, work place social support, and cardiovascular disease: A cross-sectional study of a random sample of the Swedish working population. Am J Public Health. 1988;78(10):1336-42, http://dx.doi.org/10.2105/ AJPH.78.10.1336.

21. Shimomitsu T, Haratani T, Ohno Y, Yokoyama K, Nakamura K, Iwata N, et al. Measurement of stress. In: Kato M, editor. [The Ministry of Labour sponsored grant for the prevention of work-related illness. The 1999 Report]. Tokyo: Tokyo Medical College; 2000. p. 126-64. Japanese.

22. Kawada T, Otsuka T. Relationship between job stress, occupational position and job satisfaction using a brief job stress questionnaire (BJSQ). Work. 2011;40(4):393-9, http://dx.doi.org/10.3233/WOR-2011-1251.

23. Peter R, Siegrist J, Hallqvist J, Reuterwall C, Theorell T; The SHEEP Study Group. Psychosocial work environment and myocardial infarction: Improving risk estimation by combining two complementary job stress models in the SHEEP Study. J Epidemiol Community Health. 2002;56(4):294-300, http://dx.doi.org/10.1136/ jech.56.4.294.

24. Knutsson A, Hallquist J, Reuterwall C, Theorell T, Akerstedt T. Shiftwork and myocardial infarction: A case-control study. Occup Environ Med. 1999;56(1):46-50, http://dx.doi. org/10.1136/oem.56.1.46.

25. Karasek RA. Job content questionnaire and user's guide. Lowell: University of Massachusetts; 1985.

26. Wainwright NWJ, Surtees PG, Welch AA, Luben RN, Khaw KT, Bingham SA. Healthy lifestyle choices: Could sense of coherence aid health promotion? J Epidemiol Community Health. 2007;61(10):871-6, http://dx.doi.org/10. 1136/jech.2006.056275.

27. Kuuppelomäki M, Utriainen P. A 3 year follow-up study of health care students' sense of coherence and related smoking, drinking and physical exercise factors. Int J Nurs Stud. 2003;40(4):383-8, http://dx.doi.org/10.1016/S0020-74 89(02)00103-7.

28. Cederlund RI, Ramel E, Rosberg HE, Dahlin LB. Outcome and clinical changes in patients 3, 6,12 months after a severe or major hand injury - Can sense of coherence be an indicator for rehabilitation focus? BMC Musculoskelet Disord. 2010;11:286, http://dx.doi.org/10.1186/1471-2474-11-286.

29. Teraoka S, Hayashida N, Shinkawa T, Taira Y, Nagai-Sekitani Y, Irie S, et al. Good stress management capability is associated with lower body mass index and restful sleep in the elderly. Tohoku J Exp Med. 2013;229(1):5-10, http:// dx.doi.org/10.1620/tjem.229.5.

30. Lindfors P, Lundberg O, Lundberg U. Sense of coherence and biomarkers of health in 43-year-old women. Int J Behav Med. 2005;12(2):98-102, http://dx.doi.org/10.1207/ s15327558ijbm1202_7.

31. Agardh EE, Ahlbom A, Andersson T, Efendic S, Grill V, Hallqvist J, et al. Work stress and low sense of coherence is associated with type 2 diabetes in middle-aged Swedish women. Diabetes Care. 2003;26(3):719-24, http://dx.doi. org/10.2337/diacare.26.3.719.

32. Cohen SK, Kessler RC, Gordon LU. Measureing stress: A guide for health and social scientists. New York: Oxford University Press; 1995.

33. Albertsen K, Nielsen ML, Borg V. The Danish psychosocial work environment and symptoms of stress: The 
main, mediating and moderating role of sense of coherence. Work Stress. 2001;15(3):241-53, http://dx.doi. org $/ 10.1080 / 02678370110066562$.

34. Höge T, Büssing A. The impact of sense of coherence and negative affectivity on the work stressor-strain relationship. J Occup Health Psychol. 2004;9(3):195-205, http://dx.doi. org/10.1037/1076-8998.9.3.195.

35. Brummett BH, Babyak MA, Williams RB, Barefoot JC, Costa PT, Siegler IC. NEO personality domains and gender predict levels and trends in body mass index over 14 years during midlife. J Res Pers. 2006;40(3):222-36, http://dx.doi. org/10.1016/j.jrp.2004.12.002.

36. Niaura R, Banks SM, Ward KD, Stoney CM, Spiro A 3rd, Aldwin CM, et al. Hostility and the metabolic syndrome in older males: The normative aging study. Psychosom Med. 2000;62(1):7-16, http://dx.doi.org/10.1097/00006842200001000-00002.

37. Grundy SM. Metabolic complications of obesity. Endocrine. 2000;13(2):155-65, http://dx.doi.org/10.1385/ENDO: 13:2:155.
38. Kouvonen AM, Väänänen A, Woods SA, Heponiemi T, Koskinen A, Toppinen-Tanner S. Sense of coherence and diabetes: A prospective occupational cohort study. BMC Public Health. 2008;8:46, http://dx.doi.org/10.1186/14712458-8-46.

39. Surtees P, Wainwright N, Luben R, Khaw KT, Day N. Sense of coherence and mortality in men and women in the EPIC-Norfolk United Kingdom Prospective Cohort Study. Am J Epidemiol. 2003;158(12):1202-9, http://dx.doi. org/10.1093/aje/kwg272.

40. Nakamura H, Matsuzaki I, Sasahara S, Hatta K, Nagase H, Oshita Y, et al. Enhancement of a sense of coherence and natural killer cell activity which occurred in subjects who improved their exercise habits through health education in the workplace. J Occup Health. 2003;45(5):278-85, http:// dx.doi.org/10.1539/joh.45.278.

This work is available in Open Access model and licensed under a Creative Commons Attribution-NonCommercial 3.0 Poland License - http://creativecommons.org/ licenses/by-nc/3.0/pl/deed.en. 